\title{
A predictor of pathological complete response to neoadjuvant chemotherapy in triple-negative breast cancer patients with the DNA repair genes
}

\author{
Liang Huang ${ }^{1,2 \#}$, Guan-Tian Lang ${ }^{1,2 \#}$, Qi Liu ${ }^{2,3 \#}$, Jin-Xiu Shi ${ }^{4}$, Zhi-Ming Shao ${ }^{1,2}$, A-Yong Cao ${ }^{1,2}$ \\ ${ }^{1}$ Department of Breast Surgery, Fudan University Shanghai Cancer Center/Cancer Institute, Shanghai, China; ${ }^{2}$ Department of Oncology, Shanghai \\ Medical College, Fudan University, Shanghai, China; ${ }^{3}$ Department of Radiation Oncology, Fudan University Shanghai Cancer Center/Cancer \\ Institute, Shanghai, China; ${ }^{4}$ Department of Genetics, Shanghai-MOST Key Laboratory of Health and Disease Genomics, Chinese National Human \\ Genome Center and Shanghai Industrial Technology Institute (SITI), Shanghai, China \\ Contributions: (I) Conception and design: L Huang, AY Cao; (II) Administrative support: ZM Shao; (III) Provision of study materials or patients: \\ ZM Shao; (IV) Collection and assembly of data: GT Lang, JX Shi; (V) Data analysis and interpretation: L Huang, Q Liu, AY Cao; (VI) Manuscript \\ writing: All authors; (VII) Final approval of manuscript: All authors. \\ "These authors contributed equally to this work. \\ Correspondence to: A-Yong Cao. Breast Cancer Institute, Cancer Hospital/Cancer Institute; Department of Oncology, Shanghai Medical College, \\ Fudan University, 270 Dong'an Road, Shanghai 200032, China. Email: caoayong0309@sina.com.
}

Background: We conducted this study to investigate the prevalence of potential chemo-response-related gene mutations in triple-negative breast cancer (TNBC) patients and to evaluate the potential relationship between these gene mutations and neoadjuvant chemotherapy response in TNBC patients.

Methods: One hundred sixty-two TNBC patients in Fudan University Shanghai Cancer Center who received NAC with 4 cycles of paclitaxel and carboplatin were enrolled in this study. Fifty-six pathological complete response (pCR) patients and 56 non-pCR patients were enrolled in this retrospective study for the training set. Clinical assessments of postoperative residual tumors were performed according to Response Evaluation Criteria in Solid Tumors (RECIST) 1.1 criteria. Forty chemo-response-related genes were screened in each tumor specimen by second-generation sequencing analysis. Fifty TNBC patients who received neoadjuvant chemotherapy with paclitaxel and carboplatin were enrolled in the validation group.

Results: Fifty-seven of 112 (50.9\%) TNBCs contained at least one detected somatic mutation. As expected, TP53 mutation was the most common alteration, which was observed in $21.4 \%$ of patients. BRCA1, BRCA2, RET, PI3KCA, and PTEN mutations were each observed in $11.6 \%, 4.5 \%, 5.4 \%, 2.7 \%$ and $3.6 \%$ of all cases, respectively. No significant differences in any gene mutation frequency between pCR and non-pCR groups were identified. We found that the mutation status of 10 DNA repair genes involved in homologous recombination (HR) pathway successfully discriminated between responding and nonresponding tumors in the training group. Up to 18 patients who were mutation-positive experienced pCR compared to only 6 in the non-pCR group ( $\mathrm{P}=0.006)$, and $75 \%$ the HR related gene mutation patients achieved pCR. In the validation group, TNBC patients with DNA repair gene mutations achieved $77.8 \%$ pCR.

Conclusions: A subset of TNBC patients carry deleterious somatic mutations in 10 HR-related genes. The mutation status of this expanded gene panel is likely to effectively predict respond rate to neoadjuvant chemotherapy based on paclitaxel and carboplatin. Our findings need to be validated through follow-up studies in this and additional cohorts.

Keywords: Genes mutation; triple-negative breast cancer (TNBC); neoadjuvant chemotherapy; DNA repair genes

Submitted Jun 21, 2020. Accepted for publication Oct 26, 2020.

doi: $10.21037 / \mathrm{atm}-20-4852$

View this article at: http://dx.doi.org/10.21037/atm-20-4852 


\section{Introduction}

Triple-negative breast cancer (TNBC) accounts for approximately $15 \%$ of all breast cancers worldwide and is characterized by the absence of the estrogen receptor (ER), progesterone receptor (PR) and human epidermal growth factor receptor-2 (HER-2) $(1,2)$. Due to the lack of sensitivity to hormone therapy and anti-HER-2 therapy, TNBC has become one of the refractory types of breast cancer in clinical practice, and the risk of disease recurrence and metastasis is high. Currently, no appropriate molecular targets have been identified to treat TNBC due to its heterogeneity (3). There is a close relationship between tumor gene mutation and drug resistance, disease recurrence and metastasis progression. With the development of gene mutation analyses of tumor genomes, a better understanding of response of drug therapy through analysis of tumor markers is an unmet need to elucidate potential new therapeutic targets and treatment options for TNBC.

Anthracycline-/taxane-based chemotherapy remains the standard of care systemic therapy for early-stage TNBC. Although outcomes with chemotherapy are modest overall, it is evident that a subset of TNBC patients have a higher rate of pathological complete response (pCR) to neoadjuvant chemotherapy than those with other disease phenotypes. Many investigators have proposed that hereditary germline breast cancer susceptibility genes 1 (BRCA1) mutations occur in approximately $10-20 \%$ of women with stage I-III $\mathrm{TNBC}$ and play an important role in carcinogenesis and in predicting chemotherapy responsiveness in TNBC with a characteristic pattern of DNA gains and losses (4-8). Thus, $B R C A 1$-directed therapeutic approaches, such as platinum agents and poly ADP-ribose polymerase inhibitors, are being explored for the general population of TNBC (3). In the NCT01630226 clinical trial, platinum-based single drug therapy was effective for metastatic TNBC with BRCA1/2 mutation, and carriers with $B R C A 1 / 2$ mutation have a higher therapeutic response rate. However, BRCA1/2 does not predict improved disease progression free survival (PFS) or overall survival (OS). Researchers found that neoadjuvant chemotherapy regimens containing platinum-based drugs conveyed a pathological complete remission rate (pCR) of more than $60 \%$ in BRCA1 mutated breast cancer (9). As a single drug therapy or in combination with DNA damaging drugs, poly ADP-ribose polymerase (PARP) inhibitors are particularly effective in tumors with defects in DNA damage repair. One study reported that pCR of TNBC patients with BRCA1 mutation reached $56 \%$ in patients who received six courses of carboplatin, gemcitabine and iniparib. In the I-SPY2 clinical trial, pCR of TNBC patients in response to combination therapy of veliparib/carboplatin reached $52 \%$, while that of patients with chemotherapy alone reached only $26 \%$ (10).

Neoadjuvant chemotherapy for nonmetastatic TNBC produces a higher overall response rate than other breast cancer types. However, the overall survival (OS) rate in TNBC patients did not reach pCR after receiving neoadjuvant chemotherapy and was lower than in patients with non-TNBC who had residual lesions after neoadjuvant chemotherapy $(\mathrm{P}<0.0001)(11)$. To improve OS of TNBC patients, these patients should be divided into those with good response and those with poor response according to their gene mutations. Current research is trying to explore the characteristics of TNBC from the perspective of gene phenotypes and molecular medicine with the goal of identifying new potential targeted therapeutic drugs and realizing individualized treatment. We present the following study in accordance with the TRIPOD reporting checklist (available at http://dx.doi.org/10.21037/atm-20-4852).

\section{Methods}

\section{Patients and samples}

One hundred and twelve TNBC patients for the training set and 50 patients for the validation set in the Fudan University Shanghai Cancer Center were enrolled in this retrospective study from January 2012 to December 2016. Fifty TNBC patients were enrolled in the validation group from January 2017 to July 2019. Eligibility criteria included the following: (I) clinical stage II or III patients collected and evaluated by imaging after hollow needle puncture; (II) ER-negative, PR-negative and HER2-negative; (III) These patients received neoadjuvant chemotherapy treatment with 4 cycles of paclitaxel $\left(80 \mathrm{mg} / \mathrm{m}^{2}, \mathrm{~d} 1, \mathrm{~d} 8, \mathrm{~d} 15\right)$ and carboplatin (AUC $=2$ calculated using the Calvert formula, $\mathrm{d} 1, \mathrm{~d} 8, \mathrm{~d} 15)$; (IV) all enrolled patients had complete clinical and pathological records. Clinical assessments of postoperative residual tumors were performed according to RECIST1.1 criteria. This cohort was subsequently divided into pCR and non-pCR groups. pCR was defined as the absence of invasive carcinoma in the breast and lymph nodes according to the Miller \& Payne criteria (12). All patients participating in the study provided written informed consent, and study protocols were approved by the corresponding institutional ethical committees (reference 
Table 1 Patient characteristics

\begin{tabular}{lc}
\hline Characteristics & Value \\
\hline Age at diagnosis (years), median [range] & $47[20-75]$ \\
Body mass index $\left(\mathrm{kg} / \mathrm{m}^{2}\right)$ & \\
$\leq 25$ & $85(75.9 \%)$ \\
$>25$ & $27(24.1 \%)$ \\
Menopausal status & \\
Premenopause & $76(67.9 \%)$ \\
Postmenopause & $36(32.1 \%)$ \\
Clinical tumor stage & \\
cT1 & $6(5.4 \%)$ \\
cT2 & $50(44.6 \%)$ \\
cT3 & $48(42.9 \%)$ \\
cT4 & $8(7.1 \%)$ \\
Clinical nodal stage & \\
cN0 & $21(18.8 \%)$ \\
cN1 & $61(54.5 \%)$ \\
cN2 & $13(11.6 \%)$ \\
CN3 67 index, median $[\mathrm{range}]$ & $70 \%[10-90 \%]$ \\
\hline
\end{tabular}

number: 050432-4-1212B). The study was conducted in accordance with the Declaration of Helsinki (as revised in 2013). The study was approved by the institutional review board of Fudan University Shanghai Cancer Center (reference number: 050432-4-1212B) and informed consent was taken from all the patients.

\section{Chemotherapy response related gene sequencing}

Forty chemo-response-related genes were screened in each tumor specimen by next-generation sequencing. The Gentra Puregene kit (QIAGEN, Germany) was used for DNA extraction from tumor tissue cells before neoadjuvant chemotherapy. DNA purification and concentration determination were performed using a Nanodrop 2000 (Thermo Fischer Scientific, USA). The a260/280 values of all DNA samples were between 1.8 and 2.0. Next, target genes were simultaneously specifically amplified using oligonucleotide probes designed by Illumina Design Studio (Illumina). Sequencing was performed using a MiSeq NGS system (Illumina). Data were analyzed by MiSeq Reporter software with alignment to a reference genome (grch37/ hg19) to determine the type of difference, such as deletion, insertion and single nucleotide polymorphisms. The sequence analysis software was MiSeq.

\section{Statistical analysis}

General clinical and pathological characteristics, common pathological molecular indicators, and gene mutations in patients with hollow needle puncture were summarized by descriptive analysis. The relationship between different indicators and pathological remission was evaluated by Chisquare test. Multivariate logistic regression analysis was used to predict factors related to neoadjuvant chemotherapy response. Logistic regression was used to calculate the $95 \%$ confidence interval (CI) of the odds ratios (ORs) for each variable. Statistical comparison of gene mutation status between pCR and non-pCR groups was performed using SPSS software. A P value $<0.05$ was considered statistically significant. Discrimination of the nomogram was graphically shown using a receiver operator characteristic (ROC) curve and quantified using the area under the curve (AUC).

\section{Results}

\section{Overview of TNBC patients in the training group}

Here, 56 pCR patients and 56 non-pCR patients were enrolled in this retrospective study for the training set (Table 1). All patients with breast lump hollow needle puncture were confirmed as having invasive carcinoma. Immunohistochemical pathological reports evidenced the absence of ER, PR, and HER-2, and 16.1\% (18/112) of patients with $B R C A 1 / 2$ mutation were identified by DNA sequencing of tumor tissues with preoperative hollow needle puncture. After neoadjuvant chemotherapy, the entire cohort was divided into pCR (56 cases) and nonpCR (56 cases) groups. The non-pCR group included 40 PR patients, 8 SD patients and 8 PD patients. Through retrospective analysis of clinical information, 40 patients in this cohort had a family history of malignant tumor, including 10 patients with a family history of breast cancer.

In patients with a family history of malignant tumor, $22.5 \%$ exhibited TP53 mutations, while $7.5 \%$ presented with BRCA1/2 mutations. In addition, half of patients with a family history of breast cancer exhibited TP 53 mutations. 
Table 2 Odds ratios for pathological complete response according to subgroups

\begin{tabular}{|c|c|c|c|c|}
\hline Category & \multicolumn{2}{|c|}{ Univariable } & \multicolumn{2}{|c|}{ Multivariable } \\
\hline $\begin{array}{l}\text { Menopausal status } \\
\text { (premenopause vs. postmenopause) }\end{array}$ & $0.515(0.230-1.156)$ & 0.108 & $0.881(0.320-2.429)$ & 0.807 \\
\hline Body mass index ( $\left.<25 \mathrm{vs.} \geq 25 \mathrm{~kg} / \mathrm{m}^{2}\right)$ & $0.907(0.381-2.157)$ & 0.825 & $0.894(0.362-2.211)$ & 0.809 \\
\hline $\mathrm{cN}$ (cNO vs. $\mathrm{cN}+$ ) & $1.814(0.686-4.795)$ & 0.230 & $1.887(0.690-5.162)$ & 0.216 \\
\hline Ki67 ( $\leq 30 \%$ vs. $>30 \%)$ & $3.400(1.132-10.212)$ & 0.029 & $3.018(0.884-10.302)$ & 0.078 \\
\hline
\end{tabular}

Table 3 DNA repair gene mutations and neoadjuvant chemotherapy response

\begin{tabular}{lccc}
\hline $\begin{array}{l}\text { HR pathway related } \\
\text { genes mutation }\end{array}$ & $\begin{array}{c}\mathrm{pCR} \text { patients } \\
(\mathrm{n}=56)\end{array}$ & $\begin{array}{c}\text { Non-pCR } \\
\text { patients }(\mathrm{n}=56)\end{array}$ & $\mathrm{P}$ value \\
\hline PALB2 & 4 & 0 & 0.118 \\
CHEK2 & 0 & 0 & $\mathrm{NA}$ \\
BRCA2 & 4 & 1 & 0.364 \\
RAD51D & 0 & 0 & $\mathrm{NA}$ \\
BRCA1 & 8 & 5 & 0.376 \\
RAD51C & 1 & 0 & 1.000 \\
$B R I P 1$ & 1 & 0 & 1.000 \\
ATM & 0 & 0 & $\mathrm{NA}$ \\
RAD50 & 0 & 0 & $\mathrm{NA}$ \\
BARD1 & 0 & 0 & $\mathrm{NA}$ \\
HR pathway & 18 & 6 & 0.006 \\
related 10 genes & & & \\
\hline
\end{tabular}

\section{The rate of gene mutation associated with $p C R$}

Here, 40 mutated genes related to DNA repair damage or chemotherapy were detected. We first analyzed BRCA1/2 and other homologous recombinant repair genes or chemotherapy-related gene mutation rates in both groups of TNBC patients receiving neoadjuvant chemotherapy with pCR and non-pCR. In this cohort, we found that $50.9 \%$ (57/112) of 112 TNBC patients presented with at least one somatic mutation. As expected, TP53 mutation was the most common alteration, which was observed in $21.4 \%$ of patients (36 of 52). BRCA1, BRCA2, RET, PI3KCA, and PTEN mutations were present in $11.6 \%, 4.5 \%, 5.4 \%, 2.7 \%$ and $3.6 \%$ of all cases, respectively. We found no significant differences in gene mutation frequency between the pCR and non-pCR groups (Table 2).

\section{Homologous recombination (HR) pathway-related gene mutations and neoadjuvant chemotherapy response}

In previous clinical trials, partial TNBC presented with HR deficiency, and platinum drugs may benefit these patients $(13,14)$. Further, we evaluated the potential relationship between gene mutation and neoadjuvant chemotherapy response in TNBC. An independent analysis of the combination of 10 DNA repair genes (PALB2, CHEK2, BRCA2, RAD 51D, BRCA1, RAD 51C, BRIP1, ATM, RAD50 and $B A R D 1$ ), which are involved in the HR pathway was performed. We found that the mutation status of these genes, which conveys HR deficiency, successfully discriminated between pCR and non-pCR groups treated with paclitaxel and carboplatin chemotherapy. Up to 18 patients in the $\mathrm{PCR}$ group were mutation positive compared to only 6 in the non-pCR group $(\mathrm{P}=0.006)$ (Table 3). We also found a significant difference in multivariate logistic regression analysis between pCR and non-pCR groups $(\mathrm{P}=0.013)$. The DNA repair mutation panel was predictive of pCR rate in TNBC patients who received paclitaxel and platinum. In our research of the mutational subgroup, pCR rate was as high as $75 \%$.

Ten genes whose mutations are related to DNA repair damage were also detected, and 18 of 50 (36\%) patients achieved $\mathrm{pCR}$ in the validation group. Patients with DNA repair mutations exhibited a higher pCR rate $(77.8 \%$ vs. $12.5 \%, \mathrm{P}<0.001)$. DNA repair gene mutation testing achieved an AUC of 0.826 , with a sensitivity of $77.8 \%$ and a specificity of $87.5 \%$. 


\section{Discussion}

TNBC is a commonly used umbrella term for a histologic group of tumors that are vastly heterogeneous. In fact, TNBC includes a wide range of entities differing in their biology and response to chemotherapy and targeted therapies, leading to different clinical outcomes (15). No matter the stage, local recurrence or distant metastasis occurred earlier, visceral metastasis is more common than bone metastasis, brain metastasis rate is higher, and diseasefree survival and overall survival rates are reduced.

With the rapid development of next-generation sequencing (NGS), it is now possible and affordable to sequence individual genomes in a short period to identify somatic genetic alterations (16). In the era of personalized cancer treatment, large-scale genetic analysis of tumors is considered key for a better selection of appropriate anticancer therapy (17). Couch et al. assessed the frequency of mutations in 17 predisposition genes, including BRCA1 and $B R C A 2$, in a large cohort of patients with TNBC $(\mathrm{n}=1,824)$ not selected for family history of breast or ovarian cancer who were recruited through 12 studies to determine the utility of germline genetic testing in those with TNBC. Results showed that $11.2 \%$ had mutations in BRCA1 (8.5\%) and $B R C A 2$ (2.7\%) genes (7). In the Triple-Negative Breast Cancer Consortium (TNBCC), 22 common breast cancer susceptibility variants were investigated in 2,980 Caucasian women with TNBC and 4,978 healthy controls. Six single nucleotide polymorphisms (SNPs) were significantly associated with the risk of TNBC and provided convincing evidence of genetic susceptibility for TNBC (18). It is believed that $B R C A 1$ plays a critical role in error-free DNA double-strand break repair by HR, and its deficiency can result in genomic instability (19). Due to TNBC patients having relatively high $B R C A 1$ mutation rates compared to non-TNBC patients, a larger number of studies have demonstrated that a subset of TNBC patients are susceptible to DNA double strand break-inducing therapies, such as platinum agents and poly ADP-ribose polymerase inhibitors. Thus, BRCA1-directed therapeutic approaches are being explored for the treatment of TNBC (3). Further, to improve the treatment effects of TNBC, new combination chemotherapy regimens, including platinum drugs and other targeted therapy drugs, such as antiangiogenesis, poly ADP-ribose polymerase inhibitors and other small molecule inhibitors, are in multiple clinical trials in the field of neoadjuvant therapy. The addition of platinum salts to standard neoadjuvant regimens demonstrated a significant increase in pCR rates in TNBC, reaching more than 50\% (20-22).

Despite these advances, no predictive biomarkers are currently available in the clinical setting to identify TNBC patients due to their heterogeneity, although measures of identifying such markers are being aggressively pursued. Previous studies have shown that BRCA1 and BRCA2 mutations are important potential biomarkers in TNBC with platinum therapy. TP53 gene expression and TNBC patient sensitivity to cisplatin are negatively correlated, so p53 may be a potential predictor of the efficacy of platinumbased therapy for TNBC (23). In another study, $15.2 \%$ $(16 / 105)$ of 105 TNBC cases presented changes in EGFR copy number, and the mutation frequencies of KRAS, EGFR and TP53 genes were $1.9 \%, 1.0 \%$ and $31.4 \%$, respectively. PFS and OS of metastatic TNBC subgroups with VEGFA amplification were increased after bevacizumab treatment (24). The prediction of curative effects is often performed on a small sample in a retrospective study, but large sample multicenter prospective studies are urgently needed to confirm these findings further.

Biomarkers predictive of response to neoadjuvant treatment are thus of great importance in TNBC. In addition to BRCA1/2 mutations, other mutations in DNA repair genes that are likely to be found could also be predictive markers of drug therapy. There were distinct genetic mutations observed between $\mathrm{pCR}$ and the no pCR groups of TNBC patients, some of which were useful to predict the efficacy of neoadjuvant treatment. However, until now, the prevalence of somatic mutations in DNA repair genes in TNBC have not been well documented. This knowledge is necessary to guide the application of new targeted therapeutic drugs and to predict the response to drug therapy by fully understanding the genetic variation characteristics of TNBC itself. We conducted this study to investigate the prevalence of potential chemo-responserelated genes mutated in TNBC patients and to evaluate potential relationships between these gene mutations and NAC-response in TNBC patients. In pCR and no pCR groups, we used next-generation sequencing technology to analyze 40 gene mutations in TNBC patient tissues after neoadjuvant chemotherapy. A subset of TNBC patients carried a deleterious somatic mutation in $10 \mathrm{HR}$ related genes. Mutation status of this expanded gene panel are likely to effectively predict the respond rate to neoadjuvant chemotherapy based on paclitaxel and carboplatin.

This study has several limitations common to all retrospective analyses and those with a relatively small 


\section{Page 6 of 7}

number of patients. Our study did not include other regimens commonly used for neoadjuvant therapy, and our findings need to be validated through follow-up of this and additional cohorts.

\section{Acknowledgments}

Funding: This research was supported by the National Natural Science Foundation of China (81502289), the Shanghai Natural Science Foundation (19ZR1411100), Shanghai Health Commission Foundation (201940167 and 20194Y0080), Shanghai "Rising Stars of Medical Talent" Youth Development Program (Youth Medical Talents Specialist Program-Huang Liang) and AstraZeneca. The funders had no role in the study design, data collection or analysis, decision to publish, or preparation of the manuscript.

\section{Footnote}

Reporting Checklist: The authors have completed the TRIPOD reporting checklist. Available at http://dx.doi. org/10.21037/atm-20-4852

Data Sharing Statement: Available at http://dx.doi. org/10.21037/atm-20-4852

Conflicts of Interest: All authors have completed the ICMJE uniform disclosure form (available at http://dx.doi. org/10.21037/atm-20-4852). The authors have no conflicts of interest to declare.

Ethical Statement: The authors are accountable for all aspects of the work in ensuring that questions related to the accuracy or integrity of any part of the work are appropriately investigated and resolved. The study was conducted in accordance with the Declaration of Helsinki (as revised in 2013). The study was approved by the institutional review board of Fudan University Shanghai Cancer Center (reference number: 050432-4-1212B) and informed consent was taken from all the patients.

Open Access Statement: This is an Open Access article distributed in accordance with the Creative Commons Attribution-NonCommercial-NoDerivs 4.0 International License (CC BY-NC-ND 4.0), which permits the noncommercial replication and distribution of the article with the strict proviso that no changes or edits are made and the
Huang et al. A predictor of $\mathrm{pCR}$ in TNBC with the DNA repair genes

original work is properly cited (including links to both the formal publication through the relevant DOI and the license). See: https://creativecommons.org/licenses/by-nc-nd/4.0/.

\section{References}

1. Carey LA, Dees EC, Sawyer L, et al. The triple negative paradox: primary tumor chemosensitivity of breast cancer subtypes. Clin Cancer Res 2007;13:2329-34.

2. Dent R, Trudeau M, Pritchard KI, et al. Triple-negative breast cancer: clinical features and patterns of recurrence. Clin Cancer Res 2007;13:4429-34.

3. Sharma P. Update on the Treatment of Early-Stage Triple-Negative Breast Cancer. Curr Treat Options Oncol 2018;19:22.

4. Joosse SA, van Beers EH, Tielen IH, et al. Prediction of BRCA1-association in hereditary non-BRCA1/2 breast carcinomas with array-CGH. Breast Cancer Res Treat 2009;116:479-89.

5. Gonzalez-Angulo AM, Timms KM, Liu S, et al. Incidence and outcome of BRCA mutations in unselected patients with triple receptor-negative breast cancer. Clin Cancer Res 2011;17:1082-9.

6. Sharma P, Klemp JR, Kimler BF, et al. Germline BRCA mutation evaluation in a prospective triple-negative breast cancer registry: implications for hereditary breast and/or ovarian cancer syndrome testing. Breast Cancer Res Treat 2014;145:707-14.

7. Couch FJ, Hart SN, Sharma P, et al. Inherited mutations in 17 breast cancer susceptibility genes among a large triple-negative breast cancer cohort unselected for family history of breast cancer. J Clin Oncol 2015;33:304-11.

8. Hartman AR, Kaldate RR, Sailer LM, et al. Prevalence of BRCA mutations in an unselected population of triplenegative breast cancer. Cancer 2012;118:2787-95.

9. Byrski T, Huzarski T, Dent R, et al. Pathologic complete response to neoadjuvant cisplatin in BRCA1-positive breast cancer patients. Breast Cancer Res Treat 2014;147:401-5.

10. Dent RA, Lindeman GJ, Clemons M, et al. Phase I trial of the oral PARP inhibitor olaparib in combination with paclitaxel for first- or second-line treatment of patients with metastatic triple-negative breast cancer. Breast Cancer Res 2013;15:R88.

11. Liedtke C, Mazouni C, Hess KR, et al. Response to neoadjuvant therapy and long-term survival in patients with triple-negative breast cancer. J Clin Oncol 2008;26:1275-81.

12. Ogston KN, Miller ID, Payne S, et al. A new histological 
grading system to assess response of breast cancers to primary chemotherapy: prognostic significance and survival. Breast 2003;12:320-7.

13. Loibl S, Weber KE, Timms KM, et al. Survival analysis of carboplatin added to an anthracycline/taxane-based neoadjuvant chemotherapy and HRD score as predictor of response-final results from GeparSixto. Ann Oncol 2018;29:2341-7.

14. Telli ML, Jensen KC, Vinayak S, et al. Phase II Study of Gemcitabine, Carboplatin, and Iniparib As Neoadjuvant Therapy for Triple-Negative and BRCA1/2 MutationAssociated Breast Cancer With Assessment of a TumorBased Measure of Genomic Instability: PrECOG 0105. J Clin Oncol 2015;33:1895-901.

15. Santonja A, Sanchez-Munoz A, Lluch A, et al. Triple negative breast cancer subtypes and pathologic complete response rate to neoadjuvant chemotherapy. Oncotarget 2018;9:26406-16.

16. Mefford HC. Diagnostic exome sequencing--are we there yet? N Engl J Med 2012;367:1951-3.

17. Bijlsma RM, Bredenoord AL, Gadellaa-Hooijdonk CG, et al. Unsolicited findings of next-generation sequencing for tumor analysis within a Dutch consortium: clinical daily practice reconsidered. Eur J Hum Genet 2016;24:1496-500.

18. Stevens KN, Vachon CM, Lee AM, et al. Common breast cancer susceptibility loci are associated with triple-negative breast cancer. Cancer Res 2011;71:6240-9.

19. Severson TM, Wolf DM, Yau C, et al. The BRCA1ness

Cite this article as: Huang L, Lang GT, Liu Q, Shi JX, Shao ZM, Cao AY. A predictor of pathological complete response to neoadjuvant chemotherapy in triple-negative breast cancer patients with the DNA repair genes. Ann Transl Med 2021;9(4):301. doi: 10.21037/atm-20-4852 signature is associated significantly with response to PARP inhibitor treatment versus control in the I-SPY 2 randomized neoadjuvant setting. Breast Cancer Res 2017;19:99.

20. Sikov WM, Berry DA, Perou CM, et al. Impact of the addition of carboplatin and/or bevacizumab to neoadjuvant once-per-week paclitaxel followed by dosedense doxorubicin and cyclophosphamide on pathologic complete response rates in stage II to III triple-negative breast cancer: CALGB 40603 (Alliance). J Clin Oncol 2015;33:13-21.

21. von Minckwitz G, Schneeweiss A, Loibl S, et al. Neoadjuvant carboplatin in patients with triple-negative and HER2-positive early breast cancer (GeparSixto; GBG 66): a randomised phase 2 trial. Lancet Oncol 2014;15:747-56.

22. Echavarria I, Lopez-Tarruella S, Picornell A, et al. Pathological Response in a Triple-Negative Breast Cancer Cohort Treated with Neoadjuvant Carboplatin and Docetaxel According to Lehmann's Refined Classification. Clin Cancer Res 2018;24:1845-52.

23. Jin J, Zhang $W$, Ji $W$, et al. Predictive biomarkers for triple negative breast cancer treated with platinum-based chemotherapy. Cancer Biol Ther 2017;18:369-78.

24. Schneider BP, Gray RJ, Radovich M, et al. Prognostic and predictive value of tumor vascular endothelial growth factor gene amplification in metastatic breast cancer treated with paclitaxel with and without bevacizumab; results from ECOG 2100 trial. Clin Cancer Res 2013;19:1281-9. 